\title{
18 Von lokalen Projekten der Verkehrswende für automatisierten und vernetzten Verkehr lernen
}

Diskurs- und hegemonietheoretische Perspektiven auf neue Mobilitätsdienstleistungen in Niederösterreich ${ }^{1}$

Andrea Stickler

1. Einleitung

2. Politik der Verkehrswende

3. Diskurs- und hegemonietheoretische Perspektiven

4. Neue Mobilitätsdienstleistungen als Projekte der Verkehrswende 386

4.1 Stationsbasiertes E-Car-Sharing

4.2 Gemeinnützige Fahrtendienste

4.3 Anrufsammeltaxis

5. Hegemoniale Verschiebungen mit neuen Mobilitätsdienstleistungen

6. Ableitungen für den automatisierten und vernetzten Verkehr

Literatur 


\section{EINLEITUNG}

Mit diesem Beitrag werden ausgewählte Projekte im Namen der Verkehrs- oder Mobilitätswen$\mathrm{de}^{2}$ im ländlichen und suburbanen Raum betrachtet und diese in Bezug zum automatisierten und vernetzten Fahren gesetzt. Die in diesem Artikel entwickelte These ist, dass der automatisierte und vernetzte Verkehr das bestehende „System der Automobilität“ (Urry 2004) zwar entscheidend verändern kann, die konkreten Veränderungen jedoch im Zusammenhang mit lokal-spezifischen Herausforderungen und gegenwärtigen Konflikten und Kämpfen um Mobilitätssysteme betrachtet werden müssen. Analysiert werden daher lokale Mobilitätsprojekte, mit denen über eine reine Ablösung der fossilen Antriebstechnologie der Automobilität hinausgegangen und eine umfassendere Verkehrswende mit neuen Mobilitätsdienstleistungen wie Mikrosystemen im öffentlichen Verkehr (ÖV), gemeinnützige Fahrtendienste und Car-Sharing angestrebt wird.

Die Analyse bezieht sich auf eine empirische Untersuchung im Referenzraum Niederösterreich und basiert auf einem diskurs- bzw. hegemonietheoretischen Bezugsrahmen. In Abgrenzung zu anderen soziotechnischen Wandlungstheorien, mit denen stärker die Bedeutung von technologischen Nischen und deren Zusammenwirken mit etablierten Systemen bzw. Regimen untersucht werden (Geels 2012, Loorbach et al. 2017, Kemp et al. 2012), rückt die gewählte diskurs- und hegemonietheoretische Perspektive stärker die bestehenden Machtverhältnisse und Wandlungsprozesse über gesellschaftliche Zustimmung in den Mittelpunkt (Laclau/Mouffe 2000, Nonhoff 2006, Wullweber 2012). Bezugnehmend auf Antonio Gramscis Hegemoniekonzept könne eine Veränderung - und demnach auch eine Veränderung im Sinne einer Verkehrswende - erst dann erreicht werden, wenn eine gewisse Zustimmung bzw. ein Konsens zur Veränderung in der Bevölkerung vorherrschen.

Ausgehend von dieser Annahme wird empirisch untersucht, welche Projekte der Verkehrswende sich gegenwärtig in Umsetzung befinden und alternative Mobilitätslösungen abseits des privaten, fossil-betriebenen Pkw im ländlichen und suburbanen Raum in Niederösterreich bieten. Daraus können die gegenwärtigen Herausforderungen, Spannungen und Widersprüche bei der Umsetzung empirisch abgeleitet und letztlich untersucht werden, welche Gruppen oder Institutionen für eine Veränderung im Sinne der Verkehrswende bereit sind bzw. welche Mobilitätsbedürfnisse mit den neuen Mobilitätsangeboten abgedeckt werden können.

Konkret werden drei Fallbeispiele ausgewählt: die Analyse von E-Car-Sharing-Modellen, gemeinnützige Fahrtendienste sowie Anrufsammeltaxis als zentrale Mikro-ÖV-Systeme. Aus dieser Analyse wird in einem weiteren Schritt abgeleitet, welche Strukturen bei neuen Mobilitätsangeboten gegenwärtig wirken und potentiell auch im Zusammenhang mit der Automatisierung und Vernetzung des Verkehrs eine zentrale Rolle spielen werden. Dabei wird entsprechend der

1 Teile dieses Texts sind Auszug eines Dissertationsprojekts mit dem Titel „Automobilität im Umbruch? Gegenwärtige Stabilisierungen oder Transformationen der automobilen Hegemonie“, das am Institut für Raumplanung, Forschungsbereich Soziologie bzw. am future.lab der Technischen Universität entstanden ist.

2 Die Begriffe Verkehrs- und Mobilitätswende werden im politischen Diskurs meist synonym verwendet. Zu einer präziseren begrifflichen Differenzierung siehe Beitrag 4 von Manderscheid in diesem Band. 
theoretischen Annahmen nicht davon ausgegangen, dass die gegenwärtigen relativ stabilen diskursiven Strukturen künftige Handlungen eindeutig determinieren werden. Neue Entwicklungen und Brüche können jederzeit auftreten, jedoch macht die momentane Struktur bestimmte Entwicklungen wahrscheinlicher als andere. Vorerst werden jedoch in den nächsten beiden Abschnitten kurz der Hintergrund einer Politik zur Verkehrswende aufgezeigt und zugrunde liegende diskurs- bzw. hegemonietheoretische Annahmen offengelegt.

\section{POLITIK DER VERKEHRSWENDE}

Der Verkehrssektor ist in den letzten Jahren zunehmend in den Fokus der Klima-, Energie- und Umweltpolitik gerückt. Sowohl auf internationaler Ebene als auch im nationalen und regionalen Kontext werden klimapolitische Forderungen in gesetzlich verbindlichen Zielsetzungen zum Verkehr verankert ${ }^{3}$. In Österreich betonen sowohl die nationale Klima- und Energiestrategie Mission 2030 als auch der aktuell vorgelegte nationale Klima- und Energieplan (der im Sinne der europäischen Governance-Verordnung 2018/1999 von den EU-Mitgliedsstaaten auferlegt werden muss) Ziele und Maßnahmen im Bereich des Verkehrswesens und verweisen auf die hohe Bedeutung einer umwelt- und innovationsfreundlichen Verkehrs- oder Mobilitätswende $^{4}$ (BMNT/BMVIT 2018, BMNT 2019). Gleichwohl wird die Verkehrs- und Mobilitätswende zunehmend von lokalen politischen VertreterInnen als wichtig erachtet (Österreichischer Städtebund 2015). Der Begriff der Verkehrs- oder Mobilitätswende wird oftmals sehr unterschiedlich gedeutet. Im Allgemeinen ist mit dem Begriff jedoch eine Problematisierung der Treibhausgasemissionen im Verkehrssektor, insbesondere bei der fossilen Automobilität, und der Anspruch zur Reduzierung des Autoverkehrs bzw. das Ersetzen des Privatautos durch andere Modi wie öffentlichen Verkehr, neue Mobilitätsdienstleistungen, Zufußgehen oder Radverkehr verbunden. Der politische Appell nach einer Verkehrs- und Mobilitätswende ist zu einer anerkannten Forderung geworden, die zunehmend einen weitreichenden gesellschaftlichen Zuspruch erfährt.

Die konkreten Maßnahmen und Strategien, die im Namen der Verkehrswende gesetzt werden, sind jedoch hoch umstritten. Angenommen wird, dass sowohl im Personen- als auch im Güterverkehr entscheidende Hebel in Bewegung zu setzen sind, da der Verkehr im Vergleich

3 In Österreich erfolgte diese gesetzliche Verankerung überwiegend über die „Sektorziele Verkehr“ im Klimaschutzgesetz.

4 Österreich hat sich gemäß der EU-Lastenteilungsverordnung verpflichtet, bis zum Jahr 2030 die Treibhausgasemissionen um 36 Prozent gegenüber dem Jahr 2005 zu reduzieren. Diese Zielsetzung wurde auch von den Bundesländern aufgenommen. In den Sektoren Verkehr und Gebäude werden die größten Einsparungspotentiale wahrgenommen. Der Verkehr ist derzeit der emissionsstärkste Sektor mit einem Anteil von 46 Prozent der Gesamtemissionen (außerhalb des Emissionshandels). Damit das Gesamtziel bis zum Jahr 2030 erreicht werden kann, müssen die Emissionen um rund 7,2 Millionen Tonnen $\mathrm{CO}_{2}$-Äquivalent auf rund 15,7 Millionen Tonnen $\mathrm{CO}_{2}$-Äquivalent gesenkt werden. Die Treibhausgasemissionen aus dem österreichischen Verkehrssektor (ohne Luftverkehr) sind mit 23,6 Millionen Tonnen $\mathrm{CO}_{2}$-Äquivalent für das Jahr 2017 beziffert. Demnach haben die Emissionen seit dem Jahr 1990 um 73 Prozent zugenommen. Knapp zwei Drittel der Treibhausgasemissionen des Verkehrs auf der Straße entfielen im Jahr 2017 auf den Personenverkehr. 
zu anderen Sektoren aufgrund der steigenden Treibhausgasemissionen einen Problembereich darstellt, insbesondere in Ballungsräumen und entlang von Transitrouten (BMNT 2019: 6). Neue Trends wie die Sharing Economy, neue Mobilitätskonzepte insbesondere für den ländlichen Raum, aber auch die Stärkung des öffentlichen Verkehrs sowie die Förderung von aktiver Mobilität stehen im Vordergrund. Aus technologischer Sicht werden Schlüsselrollen der Elektrifizierung und Digitalisierung zugeschrieben. Diese beiden Ebenen vereinend, soll auch die Automatisierung und Vernetzung des Verkehrs im Sinne der Verkehrswende genutzt werden (BMVIT 2018).

Trotz der bereits seit mehreren Jahrzehnten geforderten Verkehrswende blieb ihre breite Durchsetzung bislang erfolglos (Schwedes 2011: 14), das Auto ist in vielen Kontexten das dominante Verkehrsmittel geblieben (Manderscheid 2014). Zudem steigen in den letzten Jahren sowohl die Treibhausgasemissionen im Verkehrssektor als auch der Fahrzeugbestand in Österreich (Statistik Austria 2020). Diese Entwicklung wirft die Frage auf, warum die Verkehrswende, obwohl sie politisch als notwendig erachtet wird, bislang wenig erfolgreich geblieben ist. Zur theoretischen Einordnung einer Politik der Verkehrswende wird im Folgenden auf diskurs- und hegemonietheoretische Perspektiven verwiesen.

\section{DISKURS- UND HEGEMONIETHEORETISCHE PERSPEKTIVEN}

Mit dem Begriff der Verkehrswende erfolgen bestimmte Verschiebungen im hegemonialen verkehrspolitischen Diskurs, wobei überwiegend ökologische Bedenken, aber auch beispielsweise das Argument der Sicherheit (siehe Beitrag 10 von Mitteregger in diesem Band) bestimmte Veränderungen im Verkehrssektor fordern. Zur theoretischen Einordnung jener Verschiebungen wird ein diskurs- und hegemonietheoretischer Referenzrahmen herangezogen und beispielhaft auf die Politik zur Verkehrswende angewandt. Die Verschränkung von diskurstheoretischen Argumentationen und Hegemonietheorie beruht oftmals maßgeblich auf den Werken von Laclau und Mouffe (Laclau/Mouffe 2000; Laclau 1990, 2002) und wurde von Autorlnnen wie Nonhoff (2006), Vey (2015) und Wullweber $(2012,2014)$ weiterentwickelt bzw. empirisch operationalisiert. Mit dem Begriff der Hegemonie ist das Vorherrschen bestimmter Artikulationsmuster oder sozialer Sinnkonstruktionen gemeint. Hegemonie ist aber kein Zustand, sondern stets in Bewegung und Resultat einer diskursiven Praxis, die auf bestimmten diskursiven Strukturen aufbaut und diese verschiebt (Nonhoff 2006: 137). Die hegemoniale Praxis ist Teil der diskursiven Struktur des politischen Raums und entwickelt sich in diesem. Die Forderung zur Verkehrswende kann als eine solche hegemoniale Praxis gedeutet werden, die die Strukturen der Automobilität auf eine spezifische Art und Weise umdeutet bzw. verschiebt.

Die gegenwärtige Automobilität kann als eine relativ stabile diskursive Formation verstanden werden ${ }^{5}$, die jedoch umkämpft ist und sich im Zuge der zunehmend stärker werdenden Forde-

5 Sozialräumliche sowie gruppenspezifische Unterschiede bei der Nutzung des Autos spielen eine entscheidende Rolle. Während in vielen Städten die Zahl jener Personen, die ein Auto besitzen, rückläufig ist, steigt in ländlichen Regionen der Autobesitz weiterhin an (VCÖ 2015). Ebenso steht der Motorisierungsgrad und damit verbunden die Stabilität der diskursiven automobilen Formation mit der Qualität von öffentlichen Verkehrsangeboten in Verbindung. Mobilitätsforschende, die mit dem Milieu-Ansatz arbeiten, haben zudem auf die soziale Differenzierung von verschiedenen Mobilitätsmustern und die Nutzung des Autos verwiesen (Dangschat 2017, Beck/Plöger 2008). 
rung nach einer Verkehrswende erneuern muss. Wenngleich die Verkehrswende im Sinne der Dekarbonisierung bislang keine Gegenhegemonie zur Automobilität hervorgebracht hat, finden sich dennoch zahlreiche politische Artikulationen, die den universellen Geltungsanspruch des Automobils anfechten oder infrage stellen (BMNT/BMVIT 2018, BMNT 2019). Neue Technologien wie die Elektromobilität, Shared Mobility bzw. Mobility as a Service sowie die Automatisierung und Vernetzung sind auf die Weiterentwicklung der fossil-betriebenen Automobilität gerichtet und stellen Verschiebungen im hegemonialen Diskurs dar. Die Forderung nach einer Verkehrswende wird zu einer wirkmächtigen politischen Vorstellung im Verkehrssektor und bildet einen fundamentalen Knotenpunkt ${ }^{6}$, der möglichst universell konnotiert wird und verschiedene Argumentationen um die Zukunft der Mobilität zusammenführt.

Abbildung 1: Verkehrswende als Knotenpunkt

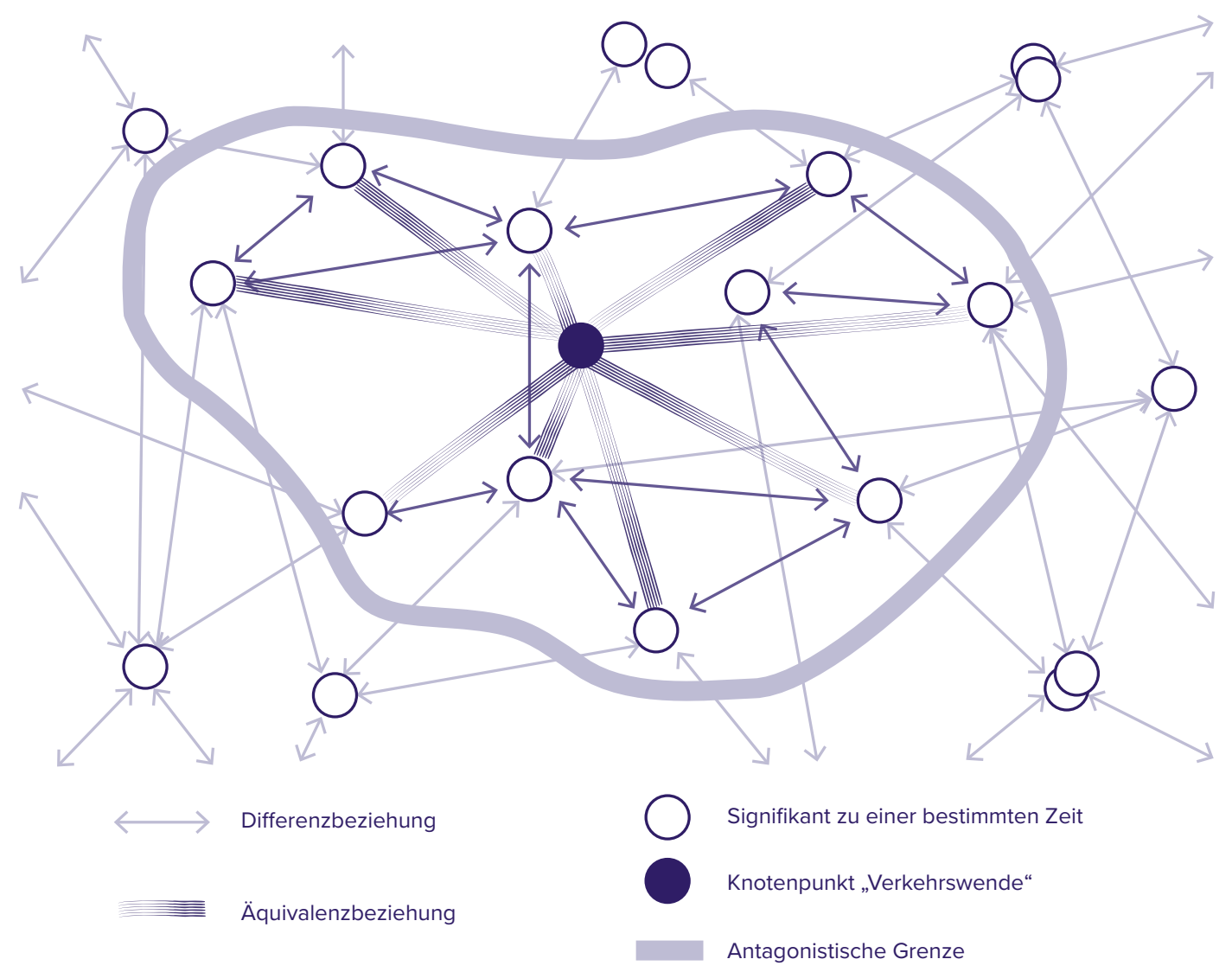

Quelle: eigene Darstellung nach Glasze (2008: 194)

6 Dieser Knotenpunkt wird in der Diskurstheorie mit dem Begriff des „leeren Signifikanten“ beschrieben. Der Begriff des Signifikanten wird auf den Sprachwissenschaftler de Saussure zurückgeführt. De Saussure untersuchte den Zusammenhang zwischen Sprache und Wirklichkeit. Zeichen bestehen aus Signifikat (dem Bedeuteten, der Vorstellung) und Signifikant (dem Bedeutenden, etwa im Falle der Sprache das Lautbild). Die Beziehung zwischen Signifikat und Signifikant ist grundsätzlich beliebig, jedoch nicht willkürlich, da die Beziehung sozial generiert wird (Hagemann 2016: 16). Durch die Universalisierung des Partikularen bei hegemonialen Projekten wird der Signifikant entleert. Das Zeichen wird von seiner Bedeutung losgelöst. 
In der gegenwärtigen Diskussion zur Verkehrswende spielen staatliche Interventionen eine entscheidende Rolle. Die aktuellen Staatsinterventionen im Sinne der Verkehrswende führen aber auch dazu, dass neue Formen der Unterordnung auferlegt werden. Beispielsweise ist die Elektromobilität trotz der hohen staatlichen Förderung gesellschaftspolitisch umstritten (Brunnengräber/Haas 2020), ebenso wie eine strukturelle (u. a. steuerliche) Benachteiligung der Automobilität. Auch die konkrete Bedeutung der Verkehrswende selbst ist umstritten. Ökologische Forderungen existieren „in vielfältigen Formen, die von der Art und Weise abhängen, in der der Antagonismus diskursiv konstituiert wird“ (Laclau/Mouffe 2000: 210). Die ökologischen Forderungen können sowohl antikapitalistisch, antiindividualistisch und autoritär als auch libertär, sozialistisch und reaktionär sein. Die Auslegung der ökologischen Forderung ist nicht von Anfang an festgelegt, sondern beweglich. Die Art und Weise wie diese Forderung artikuliert wird, ist keinesfalls vorherbestimmt.

Mit dem Begriff der Verkehrswende werden auf eine spezifische Weise äquivalente Verbindungen zu anderen großen Begriffen wie technologischem Fortschritt, Innovation, Wirtschaftswachstum, Sicherheit und Nachhaltigkeit oder erneuerbaren Energieträgern hergestellt. Durch diese begrifflichen Verbindungen (Äquivalenzbeziehungen) werden die Mängel der heutigen (Auto-)Mobilität deutlich gemacht und erhalten eine positive Präsenz (Wullweber 2014: 291). Gleichzeitig grenzt sich der Begriff der Verkehrswende von anderen großen Konzepten (wie Fossilenergie oder Zersiedelung) ab und formt dadurch eine Differenzbeziehung und antagonistische Grenze. Verschiedene gesellschaftliche Akteurlnnen verknüpfen inr Interesse mit dem Begriff der Verkehrswende. In diesem Kontext hat sich die Erzählung über Wandlungsprozesse in Richtung Elektromobilität, Sharing und Automatisierung weitgehend durchgesetzt.

Eine Reihe gesellschaftlicher Kräfte forciert also die hegemoniale Verschiebung in Richtung der Verkehrswende. Damit wird die Verkehrswende gegenwärtig erfolgreich in Verbindung mit dem gesellschaftlichen Allgemeininteresse artikuliert. Sie strebt Veränderungen an, mit denen wesentliche Herausforderungen heutiger Gesellschaften gelöst werden sollen.

\section{NEUE MOBILITÄTSDIENSTLEISTUNGEN ALS PROJEKTE DER VERKEHRSWENDE}

Die Frage, welche Verschiebungen oder Risse innerhalb des Diskurses über die Automobilität durch die Verkehrswende stattfinden, kann mittels einer genauen Betrachtung von bestehenden Projekten zur Verkehrswende beantwortet werden. Durch die Analyse von zentralen Rahmenbedingungen und geführten Gesprächen mit verschiedenen involvierten Akteurlnnen soll aufzeigt werden, welche allgemeinen Forderungen mit neuen Mobilitätsangeboten in Verbindung gebracht werden. Zudem wird untersucht, welche Faktoren für die Umsetzung und Stabilisierung der Projekte wesentlich sind bzw. wo in der Umsetzung die Herausforderungen und Probleme liegen. Ausgewählt wurden Projekte zu stationsbasiertem E-Car-Sharing, gemeinnützigen Fahrtendiensten sowie Anrufsammeltaxis. Alle analysierten Projekte liegen im suburbanen oder ländlichen Raum und zielen auf das Schaffen von alternativen Mobilitätslösungen zum Privatauto ab. Geführt wurden zwölf Gespräche mit Projektinitiatorlnnen, GemeindevertreterInnen und kommerziellen Anbietern von neuen Mobilitätsdienstleistungen. Zudem wurden Vorträge und Seminare des Landes Niederösterreich bzw. der Energie- und Umwelt-Agentur des Landes Niederösterreich hinzugezogen und ausge- 
wertet. Die Perspektiven der NutzerInnen und die breite Wahrnehmung der Angebote in der Gesellschaft konnte jedoch nicht umfassend erhoben werden und stellen einen weiteren Forschungsbedarf dar.

Die folgende Abbildung 2 liefert einen Überblick der unterschiedlichen neuen Mobilitätsangebote, die im Kontext einer Mobility as a Service genutzt werden sollen.

Abbildung 2: Mobilitätsangebote (Mobility as a Service - MaaS)

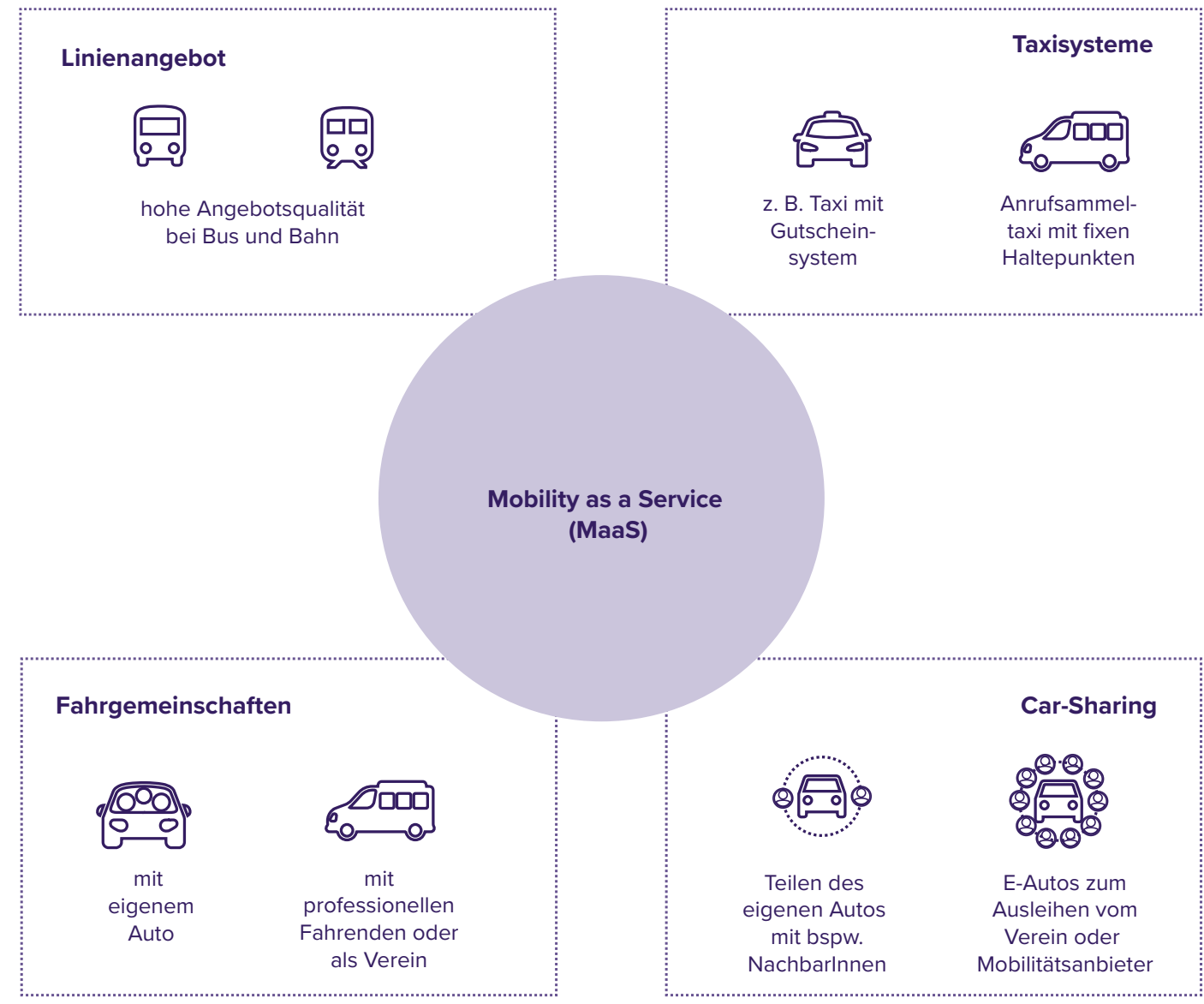

Quelle: eigene Darstellung nach Danninger (2019)

Die auf der nächsten Seite folgende Tabelle 1 vergleicht die drei ausgewählten Systeme E-CarSharing, gemeinnützige Fahrtendienste und Anrufsammeltaxis hinsichtlich der Betreiber, der Angebotsform, der Kosten für die NutzerInnen, der Art der Beförderung sowie des räumlichen Einsatzgebietes.

Mit der Analyse dieser drei unterschiedlichen Mobilitätsdienstleistungen soll untersucht werden, wie Projekte der Verkehrswende eine hegemoniale Verschiebung im relativ stabilen „System der Automobilität" (Urry 2004) erreichen können. Es stellt sich hier die Frage, ob es gelingen kann, ein Projekt so zu artikulieren, dass eine Vielzahl von Akteurlnnen die Realisierung als unerlässlich für das Allgemeininteresse ansieht und dementsprechend ihre Handlungen ausrichtet. 
Tabelle 1: Vergleich der ausgewählten Mobilitätsangebote

\begin{tabular}{|c|c|c|c|}
\hline & $\begin{array}{l}\text { Stationsgebundenes } \\
\text { E-Car-Sharing }\end{array}$ & $\begin{array}{l}\text { Gemeinnützige } \\
\text { Fahrtendienste }\end{array}$ & $\begin{array}{l}\text { Anrufsammel- } \\
\text { taxis }\end{array}$ \\
\hline $\begin{array}{l}\text { Vorwiegender } \\
\text { Betreiber }\end{array}$ & Gemeinde, Unternehmen & Verein & Gemeinde, Region \\
\hline $\begin{array}{l}\text { Vorwiegende } \\
\text { Angebotsform }\end{array}$ & $\begin{array}{c}\text { öffentlich, kommerziell } \\
\text { (mit öffentlicher Unterstützung) }\end{array}$ & $\begin{array}{c}\text { privat, nichtkommerziell } \\
\text { (mit öffentlicher Unterstützung) }\end{array}$ & $\begin{array}{c}\text { öffentlich } \\
\text { (unter Beauftragung kommerzieller } \\
\text { Transportunternehmen/ } \\
\text { Plattformbetreibern) }\end{array}$ \\
\hline $\begin{array}{l}\text { Kosten für } \\
\text { NutzerInnen }\end{array}$ & mittel & niedrig & mittel \\
\hline $\begin{array}{l}\text { Art der } \\
\text { Beförderung }\end{array}$ & $\begin{array}{l}\text { Das Fahrzeug muss selbst } \\
\text { gesteuert werden. }\end{array}$ & $\begin{array}{l}\text { Personen werden von freiwilligen } \\
\text { Fahrerlnnen befördert. }\end{array}$ & $\begin{array}{c}\text { Personen werden von } \\
\text { professionellen Fahrerlnnen } \\
\text { befördert. }\end{array}$ \\
\hline $\begin{array}{l}\text { Räumliches Einsatz- } \\
\text { gebiet }\end{array}$ & $\begin{array}{c}\text { Der Zugang erfolgt } \\
\text { stationsgebunden, das } \\
\text { Fahrzeug kann jedoch über das } \\
\text { Gemeindegebiet hinaus genutzt } \\
\text { werden. }\end{array}$ & $\begin{array}{l}\text { Der Fahrtendienst operiert } \\
\text { überwiegend innerhalb des } \\
\text { Gemeindegebietes. }\end{array}$ & $\begin{array}{l}\text { Das Anrufsammeltaxi operiert } \\
\text { in Ergänzung zum öffentlichen } \\
\text { Verkehr entlang von definierten } \\
\text { Haltepunkten. }\end{array}$ \\
\hline
\end{tabular}

Quelle: eigene Darstellung

\subsection{STATIONSBASIERTES E-CAR-SHARING}

In den letzten Jahren haben sich zahlreiche überwiegend kommunale, aber auch regionale Projekte mit der gemeinsamen Nutzung von Elektroautos im suburbanen oder ländlichen Raum beschäftigt. E-Car-Sharing ermöglicht vielen NutzerInnen, ein E-Auto einfach im Alltag zu testen und zu nutzen. Die Projekte werden oftmals zum Zweck eines Zweitwagenersatzes pro Haushalt beworben. Die Nutzungskosten für Personen, die ein Auto buchen, sind im Vergleich zu den Kosten eines eigenen Pkw relativ niedrig. Ein Imagegewinn durch innovative und nachhaltige Lösungen entsteht für den Anbieter - im ländlichen Raum ist dies meist die Gemeinde. In Niederösterreich ist der Träger des E-Car-Sharing meist ein Verein, eine Gemeinde oder auch ein professioneller Anbieter. Entscheidend ist, ob das Car-Sharing dem Gewerberecht unterliegt oder nicht.

Für das ökonomisch abgesicherte Funktionieren eines E-Car-Sharing sind maximal 20 bis 30 Nutzerlnnen pro Fahrzeug erforderlich. Sowohl Privatpersonen als auch Firmen und Gemeinden können das E-Car-Sharing initiieren. Von den Nutzerlnnen wird in der Regel eine Jahresgebühr von 100 bis 300 Euro erhoben. Zudem wird ein Kilometergeld (10 bis 20 Cent) und/oder eine Stundengebühr (1 bis 5 Euro) in Rechnung gestellt. Die Buchung des Autos erfolgt in der Regel über eine App oder ein anderes Onlinebuchungssystem. Plattformen, die eine solche Buchung ermöglichen, sind beispielsweise die Plattformen Caruso oder Ibiola. Die Buchungsplattform zeigt die Verfügbarkeit des Fahrzeuges sowie die notwendige Ladezeit zwischen den Buchungen an. Das Land Niederösterreich wird beim E-Car-Sharing als ein Vorzeigeland genannt. In mehr als 90 Gemeinden stehen über 120 E-Autos für E-Car-Sharing zur Verfügung. Zunehmend finden sich auch Projekte in Kombinationen mit E-Fahrtendiensten (Komarek 2019).

Bei E-Car-Sharing-Projekten wurde in den Beobachtungen und Befragungen deutlich, dass im ländlichen Raum vor allem in der Startphase ein starker politischer Push erforderlich ist. Es braucht eine politisch-planerisch engagierte Person, die das Thema aufgreift und in der Öffentlichkeit vertritt. So beschreibt eine befragte Person, die selbst ein E-Car-Sharing-Projekt umgesetzt hat: 
Abbildung 3: E-Car-Sharing-Projekte in Niederösterreich

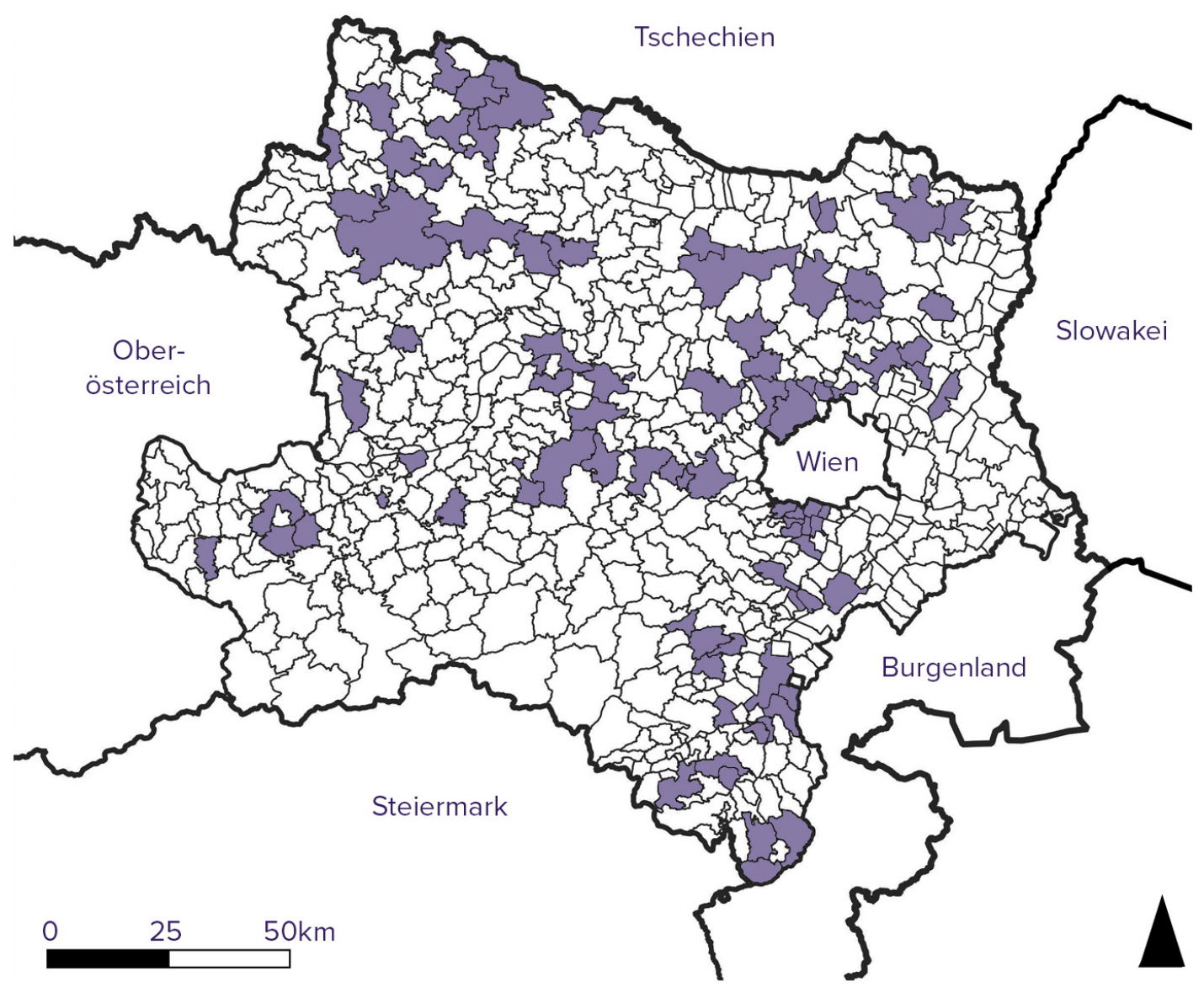

Quelle: eigene Darstellung nach eNu (2019)

„Was es meiner Meinung nach für die Umsetzung braucht: einen Kümmerer, also einen zentralen Ansprechpartner, am besten einen politischen Vertreter, der sich das Thema auf die Fahne heftet und an die Bevölkerung als tolle Sache heranträgt" (Interview A).

Die neuen Mobilitätslösungen stehen oftmals mit einem lokalpolitischen Wunsch nach Aufmerksamkeit und Innovation in Verbindung. Die Gemeinden rühmen sich mit neuen Mobilitätsprojekten und werden auch beispielsweise im Rahmen des „Clevermobil“-Wettbewerbes vom Land Niederösterreich für besonders innovative Projekte ausgezeichnet. Dieses Bestreben nach Innovationskraft zeigt sich auch in den zahlreichen Vernetzungstreffen und Seminaren, bei denen bezüglich neuer Mobilitätsdienstleistungen zwischen den politischen Vertreterlnnen der Gemeinden eine gewisse Konkurrenz und Rivalität um Innovationen spürbar ist. Die Durchsetzung von neuen Mobilitätsdienstleistungen ist zudem sehr stark vom lokalen Engagement der GemeindemitarbeiterInnen und GemeindevertreterInnen abhängig. Übergeordnete politische oder planerische Akteurlnnen geben weniger den entscheidenden Impuls zur Etablierung von E-Car-Sharing, können jedoch durchwegs auch unterstützend wirken. Mehrere Personen deuten auf die notwendige Bereitschaft der Gemeinden zum Engagement hin. Ein Projektleiter eines Car-Sharing-Projektes erwähnt in Bezug auf die Frage, wie erfolgreich der Betrieb des E-Car-Sharing derzeit ist: 
„Das hängt sehr stark vom Engagement der Gemeinden ab und wie stark die dahinter sind. Manche Gemeinden sind sehr aktiv, da funktioniert es sehr gut" (Interview B).

Diese hohe erforderliche Bereitschaft der lokalpolitischen Ebene, sich zu engagieren, wird jedoch auch durchwegs kritisch betrachtet. Vor allem im ländlichen Raum ist damit ein hohes Risiko für die initiierenden Personen verbunden. Eine weitere befragte Person, die sich für die Umsetzung eines E-Car-Sharing-Projektes eingesetzt hat (Interview E), beschreibt die Durchsetzung von E-Car-Sharing als „ständigen Kampf“, denn die Vorzüge solcher Modelle werden oftmals nicht erkannt und angenommen. Trotz der Bemühungen von lokalen politischen VertreterInnen hält sich das Interesse in der Bevölkerung für die Nutzung von E-Car-Sharing oft in Grenzen, so die Erfahrung, weshalb viele Pilotversuche nicht weitergeführt werden. In mehreren Gemeinden wurde beispielsweise in Umfragen das Interesse der Bevölkerung für E-CarSharing erhoben, jedoch blieb eine breite Rückmeldung aus. Betont wird auch das „richtige“ Zeitfenster, das es für den Start eines solchen Projektes benötigt. Zwei interviewte Gemeindevertreterlnnen beschreiben die erfolglose Startphase folgendermaßen:

„Ich habe mir 2016 [...] einen Vortrag bei der Energieagentur des Landes angehört und dann haben wir das einfach probiert [...][,] in die Gemeindezeitung gegeben und dann hat sich gerade einmal eine Person angemeldet. Danach haben wir das auch wieder fallen gelassen" (Interview H).

\begin{abstract}
"Wir haben schon soviel Zeit in ein potentielles E-Car-Sharing gesteckt und Infoveranstaltungen organisiert, bei denen sich das Interesse sehr in Grenzen gehalten hat. Ich habe schon unzählige Stunden hineininvestiert und es ist nichts daraus geworden. Bei den Infoveranstaltungen ist quasi niemand gekommen. Vielleicht waren wir auch der Zeit voraus und zu früh dran" (Interview D).
\end{abstract}

Die interviewten Gemeindevertreterlnnen beschreiben die fehlenden Interessenbekundungen auf postalische oder auch mediale Aussendungen zu einem potentiellen E-Car-Sharing-Projekt, welches die Gemeinde plante. Daraufhin folgt meist eine resignierte Haltung der politischen Vertreterlnnen und eine kritische Perspektive auf andere laufende E-Car-Sharing-Projekte. Argumentiert wird von einigen skeptischen GemeindevertreterInnen auch, dass Projekte zum E-Car-Sharing, die als erfolgreich dargestellt werden, kritisch beleuchtet werden müssen, da nicht immer die gewünschte Bevölkerungsgruppe (Autofahrende, die auf Car-Sharing umsteigen) als Nutzerlnnen eines E-Car-Sharing erreicht werde. Als herausfordernd wird auch die langfristige Bereitstellung und Nutzungskontinuität von neuen Mobilitätsservices wie E-CarSharing von den befragten Projektinitiatorlnnen genannt. So kann beispielsweise Car-Sharing auch dazu führen, dass sich Nutzerlnnen irgendwann selbst ein (E-)Auto kaufen und infolgedessen das Car-Sharing selbst kaum mehr genutzt wird.

„Eine Herausforderung ist aber auch die Kontinuität bei der Nutzung. Was passiert, wenn die Leute abspringen, weil sie sich selber ein Auto kaufen etc.? Dann gerät das System wieder ins Wanken" (Interview G).

Wenn Car-Sharing als eine solche Übergangslösung bis zur Anschaffung eines eigenen Fahrzeuges genutzt wird, dann kann Car-Sharing gegebenenfalls zu einer weiteren Stabilisierung der hegemonialen Automobilität beitragen, auch wenn diese stärker elektrisch betrieben werden könnte.

Insbesondere in Räumen, in denen die meisten Menschen ein eigenes Auto besitzen und derzeit bereits stark von der Automobilität abhängig sind, hält sich das Interesse an Car-Sharing in Grenzen. Die von GemeindevertreterInnen propagierten „Erfolgsprojekte“ werden von den 
interviewten Personen auch immer wieder kritisch eingeordnet. Aufgrund der hohen Rivalität um Innovationskraft in den Gemeinden werden viele Projekte auch überwiegend positiv dargestellt und Probleme verschwiegen. Eine befragte politische Gemeindevertreterin ordnet diese positive Darstellung kritisch ein:

\begin{abstract}
„Viele Projekte funktionieren nur, weil sie von der Gemeinde mitgenutzt werden ,müssen: Das heißt, dass eben alle Politiker und Gemeindebedienstete das Angebot nutzen müssen, was aber nicht Sinn der Sache ist. Die Projekte belügen sich mit ihrem Erfolg selbst, weil es die Bevölkerung nicht zum Umsteigen auf neue Mobilitäts/ösungen motiviert. Es müsste viel mehr aus der Bevölkerung kommen - ein Appell an die Politiker ,Hey macht das doch, wir wollen das!', aber das passiert nicht" (Interview D).
\end{abstract}

Das heißt also, dass Car-Sharing laut dieser Stellungnahme nur durch die regelmäßige Mitbenützung von GemeindemitarbeiterInnen funktioniert und damit den eigentlichen Zweck verfehlt, dass Menschen auf ein eigenes Auto verzichten und das Auto mit anderen NutzerInnen teilen. Die interviewte Person beschreibt auch die fehlenden allgemeinen Forderungen zur Durchsetzung von E-Car-Sharing aus der Bevölkerung. Trotz dieser Erfahrungen und den skeptischen Perspektiven auf E-Car-Sharing haben sie sich dennoch zunehmend in Niederösterreich etabliert. Die Car-Sharing-Projekte sind jedoch sehr unterschiedlich organisiert. Poten-

Abbildung 4: Fahrtendienste in Niederösterreich

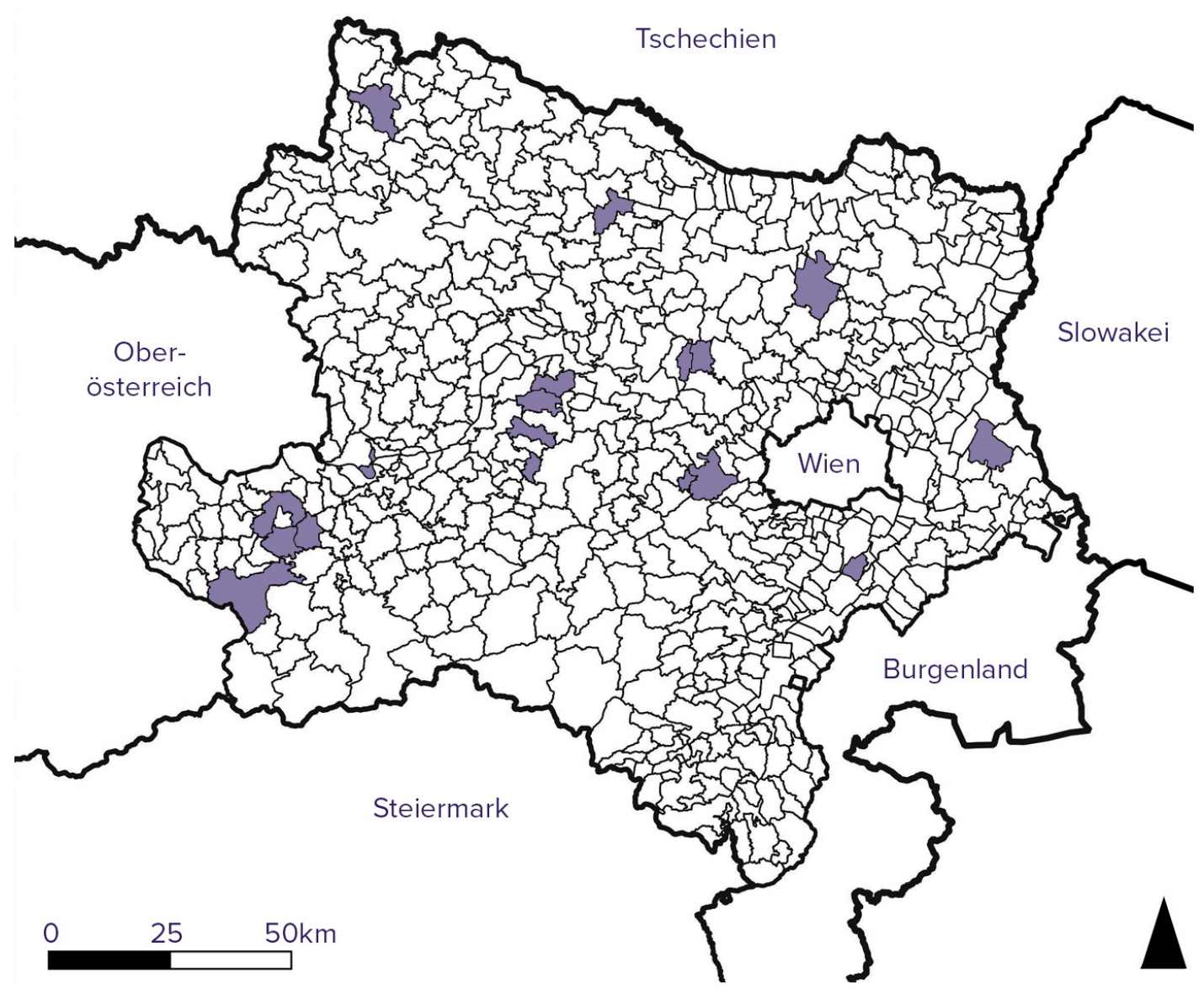


tiale für die Weiterentwicklung werden vor allem in der übergeordneten Integration in eine einheitliche Mobilitätsplattform gesehen. Aufgrund der heterogenen Anbieter- und Organisationsstrukturen erscheint dies jedoch als schwierig. Die Projekte zum E-Car-Sharing stehen demnach offensichtlich nicht immer in Verbindung mit einem gesellschaftlichen Allgemeininteresse und stellen oftmals auch keine umfassende und subsumtive Forderung zur Transformation der Automobilität. Gleichzeitig sind sie jedoch durchaus ein sehr partikularer Ausdruck von Gegenkräften zur hegemonialen Automobilität, die überwiegend durch staatliche Intervention vorangetrieben wird.

\subsection{GEMEINNÜTZIGE FAHRTENDIENSTE}

Eine weitere Nische, die im Rahmen dieses Beitrags im Sinne einer Praxis zur Verkehrswende beleuchtet wird, ist der gemeinnützige Fahrtendienst. Gemeinnützige Fahrtendienste werden mit ehrenamtlichen FahrerInnen eingerichtet und können als dekommodifizierte Mobilitätsangebote beschrieben werden: Freiwillige Fahrerlnnen befördern Vereinsmitglieder mit einem EAuto von A nach B. Dieses Modell ist als Sonderform des davor bereits bestehenden Gemeindebusses in Niederösterreich hervorgegangen. Der Gemeindebus, im Jahr 2011 entstanden, stellt eine bürgerinitiierte Beförderungsform auf lokaler Ebene dar. Ziele des Fahrtendienstes sind die Integration von weniger mobilen Personen in das Gemeindeleben sowie die Stärkung der Kommunikation und des Austausches zwischen verschiedenen Generationen. Zudem sollen bestehende Hol- und Bringfahrten von mobilitätseingeschränkten Personen (bspw. ältere Menschen oder Kinder) reduziert werden. Mit dem Fahrtendienst wird auch das Ziel der Stärkung der örtlichen Wirtschaft in Verbindung gebracht, da der Fahrtendienst auch zum Einkaufen im Ort genutzt werden soll. Im Unterschied zum E-Car-Sharing muss der Standort bei einem elektrisch betriebenen Auto nicht unmittelbar beim Wohnort der Nutzerlnnen liegen. Viele Personen können mit dem Fahrtendienst auch erste Erfahrungen mit der Elektromobilität machen, was vonseiten des Landes Niederösterreich im Sinne einer Bewusstseinsbildung als wichtig erachtet wird. Ebenso wird im Rahmen dessen die Bedeutung des Fahrtendienstes als Ersatz für den Zweitwagen in einem Haushalt diskutiert. Die Kosten für die NutzerInnen sind gering und der Fahrtendienst kann auch als Ergänzung zum öffentlichen Verkehr eingesetzt werden (Komarek 2019).

Der Träger des Fahrtendienstes ist meist ein Verein, der aber von der Gemeinde unterstützt wird. Die Kostenkalkulation erfolgt ohne Gewinnabsicht. Abzuklären ist wiederum die Gültigkeit des Gewerberechts mit der Bezirkshauptmannschaft. Wer Personen gegen Entgelt transportiert, braucht in der Regel eine Konzession (Kraftfahrlinienkonzession oder Taxi- bzw. Mietwagenkonzession). Für die Klärung der gewerberechtlichen Situation wurden auch Mustervereinsstatuten von der Niederösterreichischen Energie- und Umweltagentur ausgearbeitet und zur Verfügung gestellt. Eine Kooperation mit Taxiunternehmen und der Fahrschulen wird empfohlen: einerseits um wirtschaftliche Konkurrenzverhältnisse abzuschwächen, andererseits um das Fahrverhalten der FahrerInnen zu prüfen. Als wichtigster Erfolgsfaktor werden genügend motivierte FahrerInnen genannt. Mindestens 20 bis 30 Personen sollten hier zur Verfügung stehen. In einem weiteren Schritt sind die Betriebszeiten mit den entsprechenden Fahrschichten festzulegen. Befördert werden beim Fahrtendienst nur offiziell gelistete Vereinsmitglieder. Die Vereinsmitglieder bezahlen einen Mitgliedsbeitrag pro Monat oder pro Jahr. Meist wird dabei eine Unterscheidung zwischen ordentlichen fahrenden und außerordentlichen (mitfahrenden) Mitgliedern vorgenommen. Die Mitgliedsbeiträge können einerseits pauschal verrechnet werden, andererseits besteht zusätzlich auch die Möglichkeit, Einzelfahrtkosten abzurechnen. Organisiert wird dies über ein Buchungs-, Abrechnungs- und Administrationstool wie beispielsweise Emilio, Tullnerbacher etc. Unter bestimmten Bedingungen werden auch Fördermittel über das niederösterreichische Nahverkehrsfinanzierungsprogramm bereitgestellt (Komarek 2019). 
Der Fahrtendienst wird oftmals in Kombination mit E-Car-Sharing angewandt. In Niederösterreich waren im Jahr 2019 rund 25 gemeinnützige Fahrtendienste registriert, vor allem im ländlichen Raum (Komarek 2019). Die niederösterreichische Energie- und Umweltagentur unterstützt die Projekte zum E-Fahrtendienst vor allem in der Startphase mit einer Grobkalkulation und Umsetzungsberatung sowie einer Abendveranstaltung zur Bewerbung der Aktion und stellt Vereinsstatuten sowie ein Kommunikationspaket für die BürgerInnen zur Verfügung.

Da gemeinnützige Fahrtendienste als Vereine organisiert werden, benötigt die Gründung eines solchen Vereins hohes Engagement auf lokaler Ebene sowie persönliche Kontakte zu potentiellen Fahrerlnnen. Eine befragte Person, die selbst einen Fahrtendienst initiiert hat und in der Kommunalpolitik tätig ist, beschreibt den Gründungsprozess folgendermaßen:

„Es war ein sehr anstrengender Prozess, ich bin tagelang nur herumgefahren und habe in Kaffeehäusern nach interessierten Personen gesucht. Ich habe die Leute dort einfach angesprochen und habe 25 Mitglieder gefunden, die fix mitmachen werden. Der persönliche Kontakt war dabei sehr wichtig, dann hat sich das herumgesprochen" (Interview C).

Der Prozess beruht demnach stark auf sozialen Netzwerken und der persönlichen Kontaktaufnahme. Eine zentrale Ansprechperson ist vor allem in der Gründungsphase wichtig. Dabei ist auch zu klären, welche Personengruppen für das Projekt angesprochen werden können: Einige der Interviewten nennen als zentrale Zielgruppe jüngere PensionistInnen, aber auch junge Familien, die sich in einem neuen Umfeld engagieren und Kontakte knüpfen möchten. So beschreibt eine befragte Person der Kommunalpolitik, die selbst einen Fahrtendienst initiiert hat, den Ausgangspunkt des Fahrtendienstes wie folgt:

„Wir haben in unserer Gemeinde viele Neuzuzügler, das heißt junge Familien, die nach der Ausbildung etwa zurück aufs Land ziehen möchten. Dabei haben wir hier [eine Stadtumland-Gemeinde von Wien] eine extrem gute Lage. Von unserer Gemeinde aus erreicht man Wien in $30 \mathrm{Mi-}$ nuten, ebenso St. Pölten. Das heißt aber auch, dass viele, die in unsere Gemeinde ziehen, kaum eine größere Verwandtschaft in der Gemeinde haben. Wir haben mit dem Anrufsammeltaxi begonnen, aber das ist dann in Wirklichkeit kaum angenommen geworden. Wir haben dann ein Brainstorming gestartet und haben die Idee zu freiwilligen Fahrtendiensten entwickelt, mit E-Autos natürlich und basierend auf freiwilligen Fahrern" (Interview F).

Viele im Rahmen dieser Untersuchung befragte Personen beschreiben in den Gesprächen immer wieder Rückschläge. Nicht immer funktioniert die Umsetzung von neuen Mobilitätsdienstleistungen auf Anhieb. Während die Umsetzung eines Anrufsammeltaxis gemäß Interview $F$ vorerst nicht erfolgreich war, war der Fahrtendienst aufgrund der spezifischen sozialen Gruppe vor Ort und deren Bedürfnisse erfolgreicher. Da viele Personen kaum eine größere Verwandtschaft in der Gemeinde haben, sei die Bereitschaft für ehrenamtliches Engagement und Vereinstätigkeiten groß. Ein Fahrtendienst erfüllt nicht nur die Funktion der physischen Fortbewegung, sondern stärkt auch die Gemeinschaft unter den Mitgliedern. Die sozialen Kontakte im Verein des Fahrtendienstes erfüllen damit auch eine wichtige soziale Funktion. Dies wird von mehreren befragten Personen hervorgehoben:

„Wie ich schon erwähnt habe, ist die soziale Funktion des Ganzen wichtig. Eben weil es so herausfordernd ist, sehen es viele ältere Fahrer auch als Möglichkeit, im Alter fit zu bleiben, weil man viel Denken muss und es wichtig ist, im Alter noch eine Aufgabe zu haben" (Interview F). 
„Die Zielgruppe, die überwiegend fährt, sind jüngere Senioren, die treffen sich auch dann, um das Auto instandzuhalten, zu putzen etc. Und die Fahrer sagen mir alle, dass sie das sehr erfüllt, von daher ist es bei uns auch so ein kleines Sozialprojekt" (Interview H).

Abbildung 5: Anrufsammeltaxis in Niederösterreich

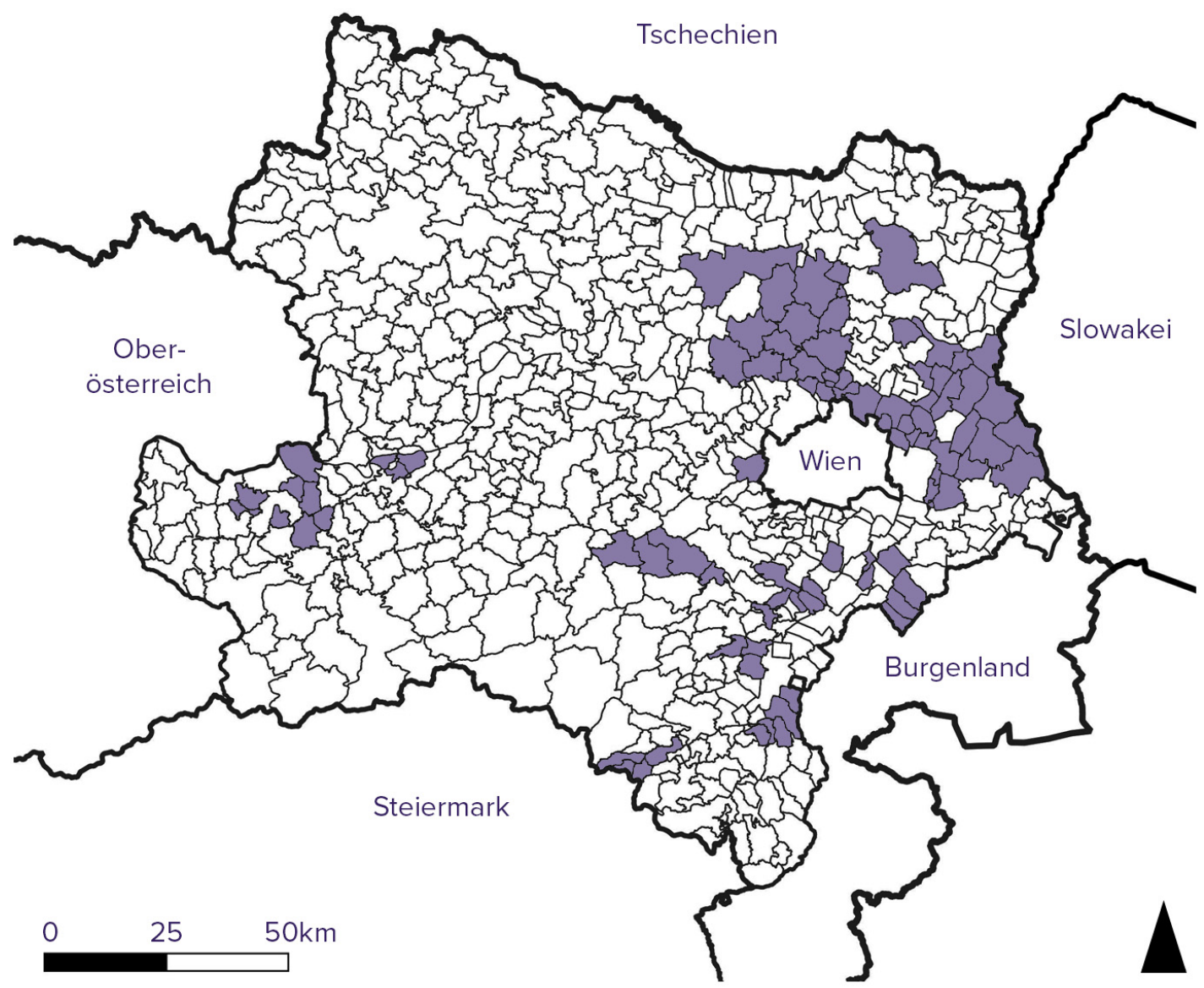

Quelle: eigene Darstellung nach Wels-Hiller (2019)

Die Betätigung in einem gemeinnützigen Fahrtendienst wird vor allem von älteren Personen als erfüllend beschrieben. Das Marketing bzw. auch das Framing des Fahrtendienstes spielt eine entscheidende Rolle. Bei einigen Fahrtendiensten wird nicht nur der geleistete Beitrag zu nachhaltiger Mobilität betont, sondern beispielsweise auch ökonomische Vorteile. Dabei nennt eine Person, dass es gerade dieses umfassende, breite Framing für den Erfolg des Fahrtendienstes braucht (Interview F). Auch der Wunsch nach medialer Aufmerksamkeit der Lokalpolitik spielt bei der Umsetzung des Fahrtendienstes eine wesentliche Rolle. Die Gemeindevertreterlnnen, aber auch die Vereinsvorstände rühmen sich mit solchen Mobilitätslösungen und werden aufgrund des hohen Innovationsgehalts von übergeordneten politischen Ebenen mit Preisen ausgezeichnet. Diese mediale Aufmerksamkeit wird in einem Interview folgendermaßen beschrieben: 
viele Nutzer, die auch was Gutes machen wollen und viele, die nachziehen" (Interview F).

Um eine größere Verbreitung und Bewerbung sowie Unterstützung des Projekts in der Bevölkerung zu erreichen, sollten gemäß mehreren befragten Personen die Gemeindevertreterlnnen im Verein vertreten sein. Oftmals ist auch der oder die Bürgermeisterln Obmann oder Obfrau des Vereins, was in der Öffentlichkeit als Signal positiv und verstärkend wirken kann. Des Weiteren spielt die Bewerbung in den regionalen oder lokalen Printmedien eine wichtige Rolle für die Ausweitung der Mitgliederanzahl.

Der Fahrtendienst richtet sich nach Auswertung der Befragung überwiegend auf eine Abkehr vom Zweitauto im Haushalt und die Integration von mobilitätseingeschränkten Personengruppen. Eine umfassende Transformation der hegemonialen Automobilität wird jedoch nicht unmittelbar angestrebt. Der Fahrtendienst spricht eine gewisse Bevölkerungsgruppe (überwiegend jüngere Seniorlnnen) an, die als Fahrerlnnen zur Verfügung stehen. Befördert werden nur gelistete Vereinsmitglieder. Damit bleibt der Fahrtendienst eine sehr partikulare Praxis. Gleichzeitig wird jedoch das gesellschaftliche Interesse aus der Bevölkerung als höher eingeschätzt als beim E-Car-Sharing. Umfassende Forderungen in Bezug auf eine weitreichende Veränderung der automobilen Hegemonie finden sich jedoch vorerst kaum im Zusammenhang mit diesen Projekten.

\subsection{ANRUFSAMMELTAXIS}

Das dritte Modell, das im Zuge dieser Analyse näher beleuchtet wird, sind Anrufsammeltaxis. Anrufsammeltaxis sowie Mikro-ÖV-Systeme im Allgemeinen dienen als Ergänzung zum öffentlichen Verkehr. Sie sollen die Flächenversorgung außerhalb des Regelverkehrs (in Ballungsräumen und frequenzstarken Regionen sowie zu Hauptverkehrszeiten) ergänzen. In frequenzschwachen Regionen und zu Schwachlastzeiten ist eine kostendeckende Bereitstellung einer Grundversorgung im öffentlichen Verkehr kaum möglich, weswegen kleinregionale, bedarfsgesteuerte Mobilitätsangebote ergänzend eingesetzt werden können.

Bei Anrufsammeltaxis werden Fahrten von Taxi- oder Mietwagenunternehmen abgewickelt. Die Fahrten können nach festgelegten Abfahrtszeiten telefonisch bestellt werden. Für die Abholung werden definierte Sammelstellen eingerichtet. Eine spezifische Linienkonzession wie im öffentlichen Verkehr ist nicht notwendig. Von der Sammelstelle können die Personen direkt zu einer konkreten Zielhaltestelle befördert werden. Der Fahrgast zahlt den regulären Tarif für den öffentlichen Verkehr mit einem Komfortzuschlag. Es erfolgt keine Beschränkung auf bestimmte Linien innerhalb des Einsatzgebiets (Gausterer 2019).

Die Vorteile des Anrufsammeltaxis sind die Bereitstellung eines wirtschaftlicheren öffentlichen Verkehrs in der Region sowie die Ergänzung zum Linienverkehr in Zeiten schwacher Nachfrage. Aufgrund der räumlichen Ungebundenheit können nicht nur spezifische Haltestellen bedient werden, sondern auch ganze Gebiete, wodurch zielgruppenspezifische Angebote geschaffen werden können. Als Nachteile werden die notwendige telefonische Voranmeldung sowie die etwas höheren Fahrpreise genannt.

Die Planung eines Anrufsammeltaxis erfordert die Definition der Zielgruppe, der Bedienzeiten sowie des Bediengebietes. Die konkrete Planung erfolgt in Abstimmung mit der Gemeinde und dem Verkehrsverbund. Die Gemeinde ermittelt dann die Kosten für den Einsatz von lokaIen Taxiunternehmen und bereitet die Verträge vor. Oftmals wird mit einer Betreiberplattform kooperiert (bspw. das Unternehmen ISTmobil). Eine Ko-Finanzierung zwischen Bund, Land und Gemeinden kann beispielsweise über das niederösterreichische Nahverkehrsfinanzie- 
rungsprogramm beantragt werden (Land Niederösterreich 2020). Bei Anrufsammeltaxis ist ein weiterer Vorteil, dass sie im Vergleich zum Fahrtendienst in keinem rechtlichen Graubereich liegen. Fahrten können auch über die Gemeindegrenze hinausgehen, sofern dies vertraglich geregelt ist. Im Weiteren ist die Mobilitätsdienstleistung nicht vom Engagement von freiwilligen FahrerInnen im Ehrenamt abhängig und keine Vereinsgründung erforderlich (Gausterer 2019). Im Vergleich zum freiwilligen Fahrtendienst können daher alle Personen befördert werden und nicht nur jene, die Mitglied in einem Verein sind.

Für eine erfolgreiche Umsetzung eines solchen Verkehrsangebots werden in den Interviews eine gezielte Steuerung und ein starkes Marketing betont. Anrufsammeltaxis sind rechtlich eindeutiger geregelt als Fahrtendienste und entstehen meist in Kooperation mit lokalen Taxiund Busunternehmen. Der Umsetzungsprozess wird als sehr zeitintensiv und anstrengend beschrieben:

„Der Prozess dahin war sehr langwierig und hat sehr viel Geduld und sehr viele Sitzungen gefordert. Ich sehe den Prozess auch noch nicht als abgeschlossen, weil es immer wieder Verbesserungen erfordert und wir das Konzept laufend verbessern" (Interview I).

Die Anrufsammeltaxis operieren an bestimmten Haltepunkten und auf bestimmten Strecken. Dabei wird auch die Konkurrenz zum öffentlichen Verkehr strategisch vermieden und nicht alle Haltepunkte angefahren. Eine befragte Person beschreibt den spezifischen Einsatzbereich des Anrufsammeltaxis folgendermaßen:

„Wir haben jetzt 634 Haltepunkte, die teilweise auch in umliegenden Gemeinden liegen, weil es so mehr Sinn macht. Wir fahren eben immer nur von Haltepunkt zu Haltepunkt und wollen keine Konkurrenz zum öffentlichen Verkehr darstellen, also haben wir es auch sehr stark auf die Zubringerfunktion zum ÖV konzipiert" (Interview I).

Die Umsetzung von Anrufsammeltaxis erfordert einen langfristigen Prozess, in dem die Haltepunkte immer wieder neu ausgehandelt werden müssen. Die Umsetzung und der Betrieb eines solchen Systems ist mit hohen Kosten für die Gemeinden verbunden ${ }^{7}$ und benötigt auch viel an rechtlicher, organisatorischer und finanzieller Unterstützung von übergeordneten politischen-planerischen Ebenen. Das Anrufsammeltaxi operiert grundsätzlich zwischen verschiedenen festgelegten Haltepunkten. Damit das Ziel der Integration von mobilitätseingeschränkten Personen erreicht werden kann, werden jedoch bestimmte Ausnahmeregelungen in Betracht gezogen:

„Wir fahren grundsätzlich nicht von Haus zu Haus, mobilitätseingeschränkte Personen können jedoch auch eine Hausabholung beantragen, die dann aber offiziell registriert werden muss" (Interview I).

Das Anrufsammeltaxi werde jedoch bei entsprechendem Angebot und ausreichender Bewerbung auch gut genutzt (Interview I). Inwiefern es auch Menschen zum Umsteigen auf den öffentlichen Verkehr motiviert, kann bislang nicht im Detail abgeschätzt werden. Die eingeschränkten Nutzungsmöglichkeiten aufgrund von vordefinierten Haltepunkten und Linien stellen jedoch im Vergleich zum eigenen Auto oder zu Fahrtendiensten einen deutlichen Nachteil dar.

7 Die anfallenden Kosten für die Gemeinden werden meist proportional zur Einwohnerlnnenzahl verrechnet. 


\section{HEGEMONIALE VERSCHIEBUNGEN MIT NEUEN MOBILITÄTSDIENSTLEISTUNGEN}

Neue Mobilitätsdienstleistungen stellen eine kongruente, aber oft sehr partikulare Lösung (hinsichtlich der Zielgruppe, der Bedienzeiten und Bediengebiete) dar, die sich in den bestehenden Diskurs zur Verkehrswende einfügt. Die Innovationen finden überwiegend innerhalb des „Systems der Automobilität" statt und stellen dieses nicht infrage, im Gegenteil: Sie versuchen die Automobilität über neue zusätzlich verfügbare Dienstleistungen neu zu definieren (nachhaltig, sozial integrativ) und damit neu zu stabilisieren. In vielen Fällen fehlen auch eine allgemeine Forderung zur Abkehr von der Automobilität sowie die gesellschaftliche Zustimmung zur weiten Verbreitung solcher Mobilitätsdienstleistungen. Daher basieren neue Mobilitätsdienstleistungen weniger auf einer aktiven Zustimmung, sondern werden vielmehr passiv geduldet und differenzieren das bestehende Mobilitätssystem aus.

Bei der Umsetzung von neuen Mobilitätsdienstleistungen werden einige Spannungsfelder deutlich. Dazu zählen beispielsweise neue wirtschaftliche Konkurrenzverhältnisse zwischen etablierten Unternehmen und neuen Mobilitätsanbietern, die bei staatlichen Interventionen strategisch vermieden werden. Durch die Absicherung von traditionellen Sektoren wie dem Taxigewerbe sowie dem öffentlichen Verkehr entstehen neue Spannungen bei der Durchsetzung von neuen Mobilitätsdienstleistungen. Ebenso problematisiert werden kann die hohe Partikularität der neuen Mobilitätsangebote, die höchst unterschiedlich organisiert sind und bislang kaum in eine übergeordnete Plattform integriert werden konnten.

Deutlich wird, dass die Projekte zu neuen Mobilitätsdienstleistungen die dominante Automobilität grundsätzlich nicht infrage stellen. Vielmehr schaffen sie ein zusätzliches Mobilitätsangebot, das im besten Falle das Zweit- oder Drittauto ablösen könnte und Menschen ohne Führerschein oder eigenem Auto mittels Auto mobilisieren kann. Neue Mobilitätsdienstleistungen sind auch oftmals noch nicht im Denken und Handeln der Menschen verankert, sondern werden durch öffentliche Interventionen im Wettbewerb um Innovation hervorgebracht. Damit können jene Mobilitätsdienstleistungen zwar als politische, jedoch nicht als hegemoniale Projekte im Sinne der Verkehrswende bezeichnet werden. Dazu fehlt das konkrete Ziel, die Reichweite und Bestimmtheit des Deutungs- und Geltungsanspruchs von neuen Mobilitätsdienstleistungen zu universalisieren und damit eine Loslösung von der hohen Abhängigkeit der gegenwärtigen Automobilität für eine breite Bevölkerungsgruppe zu bezwecken. Antagonistische Stimmen, die umfassendere Forderungen stellen und beispielsweise von spezifischen Bürgerinitiativen hervorgerbacht werden (bspw. die vernetzende Bürgerinitiative Verkehrswende Niederösterreich oder auch die Radlobby Niederösterreich), werden ins diskursive „Außen“ verschoben. Die Verkehrswende stabilisiert damit in gewissem Maße die Automobilität, wenngleich sich die Verkehrsmodi zunehmend ausdifferenzieren.

Dennoch können mit neuen Mobilitätsdienstleistungen jederzeit hegemoniale Verschiebungen stattfinden. Die Problematisierung der Automobilität im Zuge der Diskussion zur Verkehrswende eröffnet die Möglichkeit, universellere Forderungen zum Wandel der Automobilität hervorzubringen und neue Gegenprojekte dauerhaft zu fixieren und auszuweiten. Die Notwendigkeit einer Verkehrswende wird letztlich kaum mehr negiert - kritische Stimmen beanstanden nur das Wie und weniger das Ob. Die relativ stabile Automobilität ist somit keineswegs starr, sondern kann nur als diskursiv-dynamisches Phänomen dauerhaft ihre Vorherrschaft sichern. Im abschließenden Teil des Beitrags werden die potentiellen Konsequenzen dieser Erkenntnisse für den automatisierten und vernetzten Verkehr erörtert. 


\section{ABLEITUNGEN FÜR DEN AUTOMATISIERTEN UND VERNETZTEN VERKEHR}

Die Studie AVENUE21. Automatisierter Verkehr: Entwicklungen des urbanen Europa hat gezeigt, dass die räumlichen und gesellschaftlichen Wirkungen von automatisiertem und vernetztem Verkehr sehr ambivalent beurteilt werden sollten und stark von der jeweiligen politischplanerischen Steuerung (Szenario) abhängig sein werden (Mitteregger et al. 2020: 101-144). Mit dem automatisierten Fahren werden unterschiedliche Hoffnungen und Erwartungen verbunden (siehe ausführlicher in Beitrag 19 von Dangschat in diesem Band). In der Studie AVENUE21 wurde zudem ein umfassender Überblick zu den positiven und skeptischen Erwartungen im Hinblick auf den automatisierten Verkehr gegeben (ebd.: 33-45). Die positiv hervorgehobenen Aspekte sind vor allem der Rückgang der Unfallzahlen, die effiziente Steuerung der Verkehrsflüsse, eine bessere Regulierung der Geschwindigkeit und der Parkplatzsuche, die Reduktion des Energieverbrauchs und der Rückgang des Fahrzeugbestandes (und daher die Rückgewinnung von Straßenraum), die Stärkung von Intermodalität, die soziale Inklusion von mobilitätseingeschränkten Gruppen, ein stressfreies Fahren und die Nutzung der Fahrzeit für andere Tätigkeiten. Große Skepsis herrscht jedoch in Bezug auf die erwartete Verringerung des Verkehrsaufkommens und die Dekarbonisierung, denn vor allem aufgrund der neuen Bequemlichkeiten und der zunehmenden Attraktivität von peripheren Wohnstandorten, der potentiellen Erhöhung des Verkehrsaufkommens durch Leerfahrten sowie der Inklusion von neuen NutzerInnen, die bislang nicht mit dem Auto unterwegs sein konnten, ist diese Annahme anzuzweifeln (Dangschat 2019). Zudem können die Wirkungen des automatisierten Fahrens die Ziele einer nachhaltigen Siedlungsentwicklung (kompakte Stadt, Stadt der kurzen Wege, Mobilität im Umweltverbund) konterkarieren. Viele der positiven Annahmen werden erst bei einem hohen Durchdringungsgrad mit automatisierten Fahrzeugen wirksam und sind vor allem in der langen Phase des Mischverkehrs kritisch zu sehen (Mitteregger et al. 2020).

Wenn die positiven Erwartungen an die Technologie hinsichtlich der ökologischen und sozialintegrativen Forderungen in Erfüllung gehen sollen, dann sind die synergetischen Effekte der Automatisierung, Elektromobilität und des Sharing zu nutzen (ebd. 2020). Während sich Tests zum automatisierten Fahren derzeit überwiegend noch in kontrollierten Testumgebungen finden, bestehen die analysierten Mobilitätsangebote bereits seit längerer Zeit. Die analysierten Projekte verknüpfen teilweise bereits Elektromobilität und Sharing und könnten künftig auch automatisiert werden. Diese Erkenntnisse und Rückschlüsse, die sich aus gegenwärtigen Projekten der Verkehrswende ziehen lassen, können gerade deswegen hilfreich sein, weil die räumlichen, ökonomischen, ökologischen und gesellschaftlichen Wirkungen des automatisierten Fahrens hoch unsicher sind (ebd.). Aufgrund dieser allgemeinen Unsicherheit um die Automatisierung werden hier drei Aspekte aus der empirischen Fallanalyse abgeleitet und in Bezug auf den automatisierten und vernetzten Verkehr reflektiert:

- Die Durchsetzung von neuen Mobilitätsdienstleistungen im ländlichen und suburbanen Raum erfolgt derzeit sehr partikular (hinsichtlich der Zielgruppen, Bedienzeiten und Bediengebiete) und steht nicht immer in Verbindung mit umfassenden, allgemeinen Forderungen zur Transformation der Automobilität entsprechend einer gesamtheitlichen Verkehrswende. Automatisierte Fahrzeuge und Mobilitätsangebote könnten sich dementsprechend auf ähnliche Weise in diese partikularen Strukturen einfügen, ohne jedoch die Forderung einer Verkehrswende zu politisieren. Zudem ist der Charakter der Verkehrswende sehr bedeutungsoffen und kann innere Spannungen hervorbringen. Wenn Projekte zu neuen Mobilitätsdienstleistungen im Sinne einer umfassenden Verkehrswende 
eingesetzt werden sollen, dann müssten diese viel stärker das gegenwärtige automobile System infrage stellen. Erst dadurch könnten die Projekte zu neuen Mobilitätsdienstleistungen hegemonial werden und die hohe Abhängigkeit und Probleme des privaten PkwVerkehrs lösen. Wenn also neue automatisierte und vernetzte Verkehrsangebote im Sinne einer Verknüpfung von Elektromobilität, Sharing und Automatisierung eine weitreichende Durchsetzung erfahren sollen, müssten diese ebenso mit einer umfassenden Forderung zur Transformation der gegenwärtigen Automobilität in Verbindung gebracht werden und gesellschaftliche Zustimmung erfahren.

- Die gegenwärtigen Projekte zu neuen Mobilitätsdienstleistungen sind höchst unterschiedlich organisiert und strukturiert - sie gründen auf einem starken lokalen Engagement, komplexen Abstimmungsprozessen zwischen verschiedenen Akteurlnnen und verzeichnen unterschiedliche Betriebsformen, Modelle oder Zielgruppen. Die Zusammenführung dieser differenten Projekte hin zu einem gemeinsamen hegemonialen Projekt der Verkehrswende stellt ebenso eine große Herausforderung dar wie eine umfassende Integration der Angebote im Sinne von Mobility as a Service. Die Durchsetzung des automatisierten und vernetzten Verkehrs wird daher entsprechend der heutigen Projekte umkämpft sein und zahlreiche Abstimmungen bzw. Aushandlungen erfordern. Es kann somit von keinem linearen, konfliktfreien oder hoheitlich gesteuerten Wandel ausgegangen werden. Ein Wandel im Sinne der Verkehrswende benötigt entsprechend der hegemonietheoretischen Perspektive vielmehr die Zustimmung der Bevölkerung. Der Bevölkerung muss dabei auch Gestaltungsmacht zugesprochen werden.

- Eine Durchsetzung von kollektiven, geteilten oder öffentlichen Verkehrsangeboten erfordert zudem einen breiteren Wertewandel. Dieser Wertewandel kann über Erfahrungen mit den neuen Mobilitätslösungen angestoßen werden: Fahrtendienste, Anrufsammeltaxis, Car-Sharing, aber auch Testumgebungen zum automatisierten und vernetzten Fahren können die Vorteile von diesen Systemen im Vergleich zur gegenwärtigen Automobilität (Kosten, Instandhaltung, Platzverbrauch, Emissionen etc.) erfahrbar machen und einen Wertewandel begünstigen. Diesbezüglich können die heutigen Projekte zu neuen Mobilitätsdienstleistungen auch antagonistische Kräfte zur hegemonialen Automobilität stärken. Während jedoch die sozialen Funktionen von gemeinnützigen Fahrtendiensten mit der Automatisierung potentiell eher entfallen, können Kostenvorteile für Anrufsammeltaxis aufgrund des Wegfalls von Betriebskosten tendenziell eher erreicht werden. Stationsbasiertes Car-Sharing könnte zunehmend auch stationsungebunden und automatisiert funktionieren. Wenn durch die Automatisierung in bestimmten Umgebungen keine Fahrzeuglenkung mehr notwendig ist, dann kann Car-Sharing auch zunehmend mit Taxissystemen oder Fahrtendiensten konvergieren. Eine Automatisierung und Vernetzung des Verkehrs kann damit auf diesen Wertewandel hin zur breiten Akzeptanz von geteilten Verkehrsangeboten, u. a. auch aufgrund der allgemeinen Faszination um technologische Neuerungen, positiv wirken.

\section{LITERATUR}

Beck, S., und W. Plöger 2008. „Lebensstile und Mobilität“, in Stadtentwicklung. Lebensstile und Mobilität. Bundesverband für Wohnen und Stadtentwicklung, vhw FW 1, 48-51. https://tinyurl.com/ y82e6zmn (23.4.2020).

BMNT (Bundesministerium für Nachhaltigkeit und Tourismus) 2019. „Entwurf des integrierten nationaIen Energie- und Klimaplans für Österreich. Periode 2021-2030“, 18.12.2019. Wien. 
BMNT (Bundesministerium für Nachhaltigkeit und Tourismus) und BMVIT (Bundesministerium für Verkehr, Innovation und Technologie) 2018. „Mission 2030 - Die österreichische Klima- und Energiestrategie“. www.mission2030.bmnt.gv.at (2.4.2019).

BMVIT (Bundesministerium für Verkehr, Innovation und Technologie) 2018. „Aktionspaket automatisierte Mobilität 2019-2022“. Wien. https://mobilitaetderzukunft.at/resources/pdf/broschueren/aktionspakte-automatisierte-mobilitaet-2019.pdf (23.4.2020).

Brunnengräber, A. und Haas, T. 2020. Baustelle Elektromobilität. Sozialwissenschaftliche Perspektiven auf die Transformation der (Auto-)Mobilität. Berlin: Transkript.

Dangschat, J. S. 2017. „Wie bewegen sich die (Im-)Mobilen? Ein Beitrag zur Weiterentwicklung der Mobilitätsgenese", in Verkehr und Mobilität zwischen Alltagspraxis und Planungstheorie. Ökologische und soziale Perspektiven, hg. v. M. Wilde, M. Gather, C. Neiberger und J. Scheiner. Wiesbaden: Springer VS, 25-52.

Dangschat, J. S. 2019. „Automatisierte und vernetzte Fahrzeuge - Trojanische Pferde der Digitalisierung?", in Infrastruktur und Mobilität in Zeiten des Klimawandels, Jahrbuch Raumplanung Band 6, hg. v. M. Berger, J. Forster, M. Getzner und P. Hirschler (Hrsg.). Wien: Neuer Wissenschaftlicher Verlag, 11-28.

Danninger, O. 2019. „Dekarbonisierung der Mobilität in NÖ“, Vortrag im Rahmen der 5. Fachtagung „e-mobil in niederösterreich“ in St. Pölten. www.ecoplus.at/media/14895/1_danninger_dekarbonisierung.pdf (30.1.2020).

eNu (Energie- und Umweltagentur des Landes NÖ) 2019. „Projekte in Niederösterreich“, www.umweltgemeinde.at/e-carsharing-in-niederoesterreich (31.1.2020).

Gausterer, F. 2019. „Projektabwicklung und Empfehlungen für Trägerorganisationen“, Vortrag im Rahmen des Seminars „E-Fahrtendienst in NÖ“ der eNu (Energie- und Umweltagentur des Landes NÖ), 6.11.2019, Pölten.

Geels, F. W. 2012. „A socio-technical analysis of low-carbon transitions: introducing the multi-level perspective into transport studies“, in Journal of Transport Geography 24, 471-482.

Glasze, G. 2008. „Vorschläge zur Operationalisierung der Diskurstheorie von Laclau und Mouffe in einer Triangulation von lexikometrischen und interpretativen Methoden“, in Historical Social Research (33) 1, 185-223.

Hagemann, I. 2016. „Das gegenhegemoniale Moment der Demokratie. Gegenhegemoniale Projekte und demokratische Demokratie am Fallbeispiel der grünen Bewegung“, Dissertation, Universität Duisburg-Essen.

Kemp, R., F. W. Geels und G. Dudley 2012. „Introduction“, in Automobility in Transition? A Socio-Technical Analysis of Sustainable Transport, hg. v. F. W. Geels, R. Kemp, G. Dudley und G. Lyons. New York/London: Routledge, 3-28.

Komarek, M. 2019. „e-Mobilität \& e-Fahrtendienst in NÖ“, Vortrag im Rahmen des Seminars „E-Fahrtendienst in NÖ“ der eNu (Energie- und Umweltagentur des Landes NÖ), 6.11.2019, St. Pölten.

Laclau, E. 1990. New Reflections on the revolution of our time. London: Verso.

Laclau, E. 2002. „Was haben leere Signifikanten mit Politik zu tun?“, in Emanzipation und Differenz, hg. v. E. Laclau. Wien: Turia \& Kant, 65-78.

Laclau, E. und C. Mouffe 2000. Hegemonie und radikale Demokratie. Zur Dekonstruktion des Marxismus. Wien: Passagen.

Land Niederösterreich 2020. „NÖ Nahverkehrsfinanzierungsprogramm (NÖ NVFP)“, http://www.noe. gv.at/noe/OeffentlicherVerkehr/Foerd_NOE_NVFP.html (1.2.2020).

Loorbach, D., N. Frantzekaki und F. Avelino 2017. „Sustainability Transitions Research: Transforming Science and Practice for Societal Change“, in Annual Review of Environment and Resources (42) 1, 599-626.

Manderscheid, K. 2014. „Formierung und Wandel hegemonialer Mobilitätsdispositive: Automobile Subjekte und urbane Nomaden“, in Zeitschrift für Diskursforschung 2/1, 5-31.

Mitteregger, M., E. M. Bruck, A. Soteropoulos, A. Stickler, M. Berger, J. S. Dangschat, R. Scheuvens und I. Banerjee 2020. AVENUE21. Automatisierter und vernetzter Verkehr: Entwicklungen des urbanen Europa. Berlin: Springer Vieweg.

Nonhoff, M. 2006. Politischer Diskurs und Hegemonie. Das Projekt „Soziale Marktwirtschaft“. Bielefeld: transcript. 
Österreichischer Städtebund 2015. „Positionspapier des Städtebundes zum bundesweiten Handlungsbedarf im Bereich des städtischen/stadtregionalen ÖPNV“, 18.3.2015.

Schwedes, Oliver 2011. „Statt einer Einleitung“, in Verkehrspolitik. Eine interdisziplinäre Einführung, hg. v. O. Schwedes. Wiesbaden: VS Verlag, 13-36.

Statistik Austria 2020. „Pressemitteilung: 12.165-005/20. Pkw-Neuzulassungen“. www.statistik.at/ web_de/presse/122440.html (15.1.2020).

Urry, John 2004. „The ,System“ of Automobility“, in Theory, Culture \& Society 21 (4/5), 25-39.

VCÖ (Verkehrsclub Österreich) 2015. „Beim Autobesitz geht Schere zwischen Stadt und Land immer weiter auseinander". www.vcoe.at/presse/presseaussendungen/detail/vcoe-beim-autobesitzgeht-schere-zwischen-stadt-und-land-immer-weiter-auseinander (26.3.2020).

Vey, J. 2015. Gegen-hegemoniale Perspektiven. Analyse linker Krisenproteste in Deutschland 2009/2010. Hamburg: VSA. https://tinyurl.com/ya5vz95f (23.4.2020).

Wels-Hiller, S. 2019. „NÖ Gemeindebus-Modell. Grundlagen und Fördermöglichkeiten.“ Vortrag im Rahmen des Seminars „E-Fahrtendienst in NÖ“ der eNu (Energie- und Umweltagentur des Landes NÖ), 6.11.2019, St. Pölten.

Wullweber, J. 2012. „Konturen eines politischen Analyserahmens - Hegemonie, Diskurs und Antagonismus“, in Diskurs und Hegemonie. Gesellschaftskritische Perspektiven, hg. v. I. Dzudzek, C. Kunze und J. Wullweber. Bielefeld: transcript, 29-58.

Wullweber, J. 2014. „Leere Signifikanten, hegemoniale Projekte und internationale Innovations- und Nanotechnologiepolitik“, in Diskursforschung in den Internationalen Beziehungen, hg. v. E. Herschinger und J. Renner. Baden-Baden: Nomos, 270-306. 
Open Access Dieser Beitrag wird unter der Creative Commons Namensnennung 4.0 International Lizenz (http://creativecommons.org/licenses/by/4.0/deed.de) veröffentlicht, welche die Nutzung, Vervielfältigung, Bearbeitung, Verbreitung und Wiedergabe in jeglichem Medium und Format erlaubt, sofern Sie den/die ursprünglichen Autor(en) und die Quelle ordnungsgemäß nennen, einen Link zur Creative Commons Lizenz beifügen und angeben, ob Änderungen vorgenommen wurden.

Die in diesem Beitrag enthaltenen Bilder und sonstiges Drittmaterial unterliegen ebenfalls der genannten Creative Commons Lizenz, sofern sich aus der Abbildungslegende nichts anderes ergibt. Sofern das betreffende Material nicht unter der genannten Creative Commons Lizenz steht und die betreffende Handlung nicht nach gesetzlichen Vorschriften erlaubt ist, ist für die oben aufgeführten Weiterverwendungen des Materials die Einwilligung des jeweiligen Rechteinhabers einzuholen.

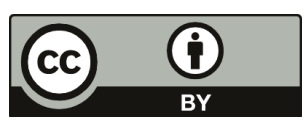

